\title{
Virtual Gynaeoncology Clinics: Are they an effective way to keep the cancer pathway working in the COVID-19 era? A Commentary
}

\author{
Thumuluru Kavitha Madhuri ${ }^{1}$ and Alex $\operatorname{Tan}^{2}$ \\ ${ }^{1}$ Royal Surrey County Hospital NHS Foundation Trust \\ ${ }^{2}$ Royal Surrey NHS Foundation Trust
}

July 3, 2020

\begin{abstract}
The COVID-19 pandemic has presented the NHS with unprecedented challenges following the declaration of "global pandemic" by the WHO. ${ }^{1}$ In response to this crisis, reconfiguration of services and resource allocation were undertaken across all levels with a view to provide effective supportive and emergency care as well as minimise risk of exposure to the infection in hospitals. ${ }^{1}$
\end{abstract}

The COVID-19 pandemic has presented the NHS with unprecedented challenges following the declaration of "global pandemic" by the WHO. ${ }^{1}$ In response to this crisis, reconfiguration of services and resource allocation were undertaken across all levels with a view to provide effective supportive and emergency care as well as minimise risk of exposure to the infection in hospitals. ${ }^{1}$

As an immediate adaptation, the NHS reduced face-to-face outpatient consultations and postponed nonurgent appointments. ${ }^{2,3}$ This was believed to be a pragmatic approach in reducing footfall into hospitals and primary care providers which, in turn, would reduce viral transmission in the outpatient setting. ${ }^{2,3}$

The cost of this transient process has been significant to stakeholders and while the true impact of this reaction is yet to be fully revealed, patients requiring key investigations and treatment in secondary care have been hesitant to seek help for two common reasons. ${ }^{4,5}$ This was mainly due to patients not wanting to be a burden on healthcare providers during this crisis, drawing them away from sick patients and the ongoing fear and anxiety of contracting the viral infection within the hospital. ${ }^{4,5}$

The NHS quarterly results (Q2 2019/2020) reported that the number of women seen with a suspected gynaecological malignancy within the two-week wait and diagnosed (31-day target) ranges widely across the UK regions. ${ }^{6}$ Rapid adoption of telehealth and telemedicine allowed the NHS to explore and employ virtual modes of consultations. ${ }^{7}$ These virtual clinics would typically involve a video conference or telephone consultation between the healthcare professional and patient on registered contact details to discuss various aspects of their care, ranging from triaging reported symptoms, alleviating anxiety to facilitating key investigations and expediting management plans. ${ }^{5,7}$

As early adopters of the virtual clinic model in gynaecological oncology, we would encourage all departments to allocate designated time to assess new cancer referrals as well as follow up patients under surveillance and effectively screen for individuals most likely to benefit from this approach rather than deferring or cancelling appointments as the initial response. Where feasible and with patient consent, they are booked in for a virtual consultation using existing trust models of secure electronic communication and contacted with clear instructions via text, email or post depending on local trust policy.

Digitisation of patient records through electronic patient records (EPR) remains key to enable NHS delivery of such virtual clinics. ${ }^{8.9}$ In light of the increased use of various commercial platforms including Zoom and 
Microsoft Teams for both clinical and managerial activities, safer data encrypted platforms such as NHS attend anywhere platform should be advocated for all NHS healthcare professionals in order to be compliant with the Data Protection Act and Patient Confidentiality. ${ }^{10}$

All virtual consultations should be ideally recorded and clearly documented in case notes. An audit trail of written correspondence between the secondary care professionals, primary care providers and patients should be regularly maintained in the similar manner as conventional face-to-face consultations in order to maintain continuity of care.

Despite playing a major role across most specialties in this crisis, we do not feel that virtual clinics are superior to face-to-face consultations as the gold standard of care. Certain individuals with a high index of suspicion of malignancy are still encouraged to attend hospital appointments for a timely diagnosis and early treatment. We also need to seriously consider the ethical implications and moral dilemmas of discussing new diagnoses, complex treatment regimens and cancer follow-ups virtually, especially in vulnerable patients. However, we acknowledge the acceptability of this modified approach based on various patient satisfaction surveys and its future role in advocating patient-initiated follow up services in selected groups of individuals. ${ }^{11}$

Despite facing numerous limitations in the delivery of our cancer care, an opportunity for change has presented itself and the gynaecological oncology and other allied health professional teams could embrace this challenge as a cohesive team and actively implement targeted virtual clinics to advocate timely diagnosis and expedite treatment.

Disclosure of interests: Both authors confirm that they have no interests to declare

Contribution to authorship: TKM conceived the idea and both TKM and AT contributed to the manuscript preparation. Both authors have read and approved the final draft submitted.

\section{Ethics Approval: Not required}

\section{Funding: none}

Pandemics pose a challenge to all involved in the delivery of healthcare; however, using a targeted virtual clinic approach will keep the pathway moving for patients with high-risk urological malignancies and enable us to resume services in a much-improved position NHS England. NHS Statistics on Waiting Times for Suspected and Diagnosed Cancer Patients Q2 2019/20. NHS Englan

\section{References}

1. WHO Coronavirus Disease (COVID-19) Dashboard. World Health Organization. Webpage. Available from https://covid19.who.int/ [Accessed 24 ${ }^{\text {th }}$ June 2020]

2. NHS long term plan. NHS. Webpage. Available from https://www.longtermplan.nhs.uk/ [Accessed 24th June 2020]

3. Van de Haar J, Hoes LR, Coles CE, et al. Caring for patients with cancer in the COVID-19 era. Nat Med 2020 May; 26(5):665-671. Doi: 10.1038/s41591-020-0874-8. Epub 2020 Apr 16.

4. Coronavirus and the impact on output in the UK economy: April 2020.Office for National Statistics. Webpage. Available from https://www.ons.gov.uk/economy/grossdomesticproductgdp/articles/coronavirusandtheimpactonoutputintheukeconon [Accessed 24 ${ }^{\text {th }}$ June 2020]

5. Rodler S, Apfelbeck M, Schulz GB, et al. Telehealth in Uro-Oncology beyond the pandemic: Toll or Lifesaver? Eur Urol Focus. 2020 Jun 10;S2405-4569(20)30145-0. Doi:10.1016/j.euf.2020.05.010.

6. Quarterly Provider Based Cancer Waiting Time Statistics. NHS.Webpage. Available from https://www.england.nhs.uk/statistics/statistical-work-areas/cancer-waiting-times/quarterly-provcwt/ [Accessed 24 ${ }^{\text {th }}$ June 2020]

7. Al-Shamsi HO, Alhazzani W, Alhuraiji A, et al. A practical approach to the management of cancer patients during the noval coronavirus disease 2019 (COVID-19) pandemic: An international collaborative group. Oncologist. 2020 Jun;25(6):e936-945. Doi: 10.1634/theoncologist.2020-0213. Epub 2020 
Apr 27.

8. Hollander JE, Carr BG. Virtually Perfect? Telemedicine for COVID-19. $N$ Engl J Med. 2020 Apr 30;382(18):1679-1681. Doi: 10.1056/NEJMp2003539. Epub 2020 Mar 11.

9. Hollins L-Transforming outpatient care. NHS. Weblog. Available from https://www.england.nhs.uk/blog/transforming-outpatient-care/ [Accessed $24^{\text {th }}$ June 2020]

10. What is Attend Anywhere/Near Me?. NHS Scotland. Webpage, Available from https://www.vc.scot.nhs.uk/attendanywhere/ [Accessed 24 ${ }^{\text {th }}$ June 2020]

11. Newton C, Nordin A, Rolland P, et al. British Gynaecological Cancer Society Recommendations and Guidance on Patient-Initiated Follow-Up (PIFU). Int J Gynecol Cancer. 2020 May; 30(5):695-700. Doi: 10.1136/ijgc-2019-001176.Epub Apr 19. 